\title{
598.
}

\section{NOTE ON A PROCESS OF INTEGRATION.}

[From the Messenger of Mathematics, vol. Iv. (1875), pp. 149, 150.]

I HAD occasion to consider the integral

$$
\int_{0}^{R} \frac{r^{s-1} d r}{\left\{r^{2}+e^{2}\right\}^{\frac{1}{2} s+q}}
$$

where $e$ is small in regard to $R$ and $q$ is negative. The integral is finite when $e=0$, and it might be imagined that it could be expanded in positive powers of $e$; and, assuming it to be thus expansible, that the process would simply be to expand under the integral sign in ascending powers of $e$, and integrate each term separately, so that the series would be in integer powers of $e^{2}$.

Take two particular cases. First, let

the integral is

$$
s=2, \quad q=-\frac{3}{2}
$$

$$
\begin{aligned}
\int_{0}^{R} r \sqrt{ }\left(r^{2}+e^{2}\right) d r & =\int_{0}^{R} d r\left(r^{2}+\frac{1}{2} e^{2} r^{0}-\frac{1}{8} e^{4} r^{-2}+\ldots\right) \\
& =\frac{1}{3} R^{3}+\frac{1}{2} e^{2} R+\infty e^{4}+\ldots
\end{aligned}
$$

viz. the integral is not thus obtainable: the series is right as far as it goes, but the true expansion contains a term in $e^{3}$; and the failure of the series to give the true expansion is indicated by the appearance of infinite coefficients. In fact, the indefinite integral is $\frac{1}{3}\left(r^{2}+e^{2}\right)^{\frac{3}{2}}$; taking this between the limits, it is

$$
\frac{1}{3}\left(R^{2}+e^{2}\right)^{\frac{3}{2}}-\frac{1}{3} e^{3}, \quad=\frac{1}{3} R^{3}+\frac{1}{2} e^{2} R+\ldots-\frac{1}{3} e^{3} .
$$

Again, let $s=1, q=-2$; the integral is

$$
\begin{aligned}
\int_{0}^{R}\left(r^{2}+e^{2}\right)^{\frac{8}{2}} d r & =\int_{0}^{R}\left(r^{2}+\frac{3}{2} e^{2} r+\frac{3}{8} e^{4} r^{-1}+\ldots\right) \\
& =\frac{1}{4} R^{4}+\frac{3}{4} e^{2} R^{2}+\infty e^{4}+\ldots
\end{aligned}
$$

C. IX. 
viz. the integral is not thus obtainable: the series is right as far as it goes, but the true expansion contains a term as $e^{4} \log e$, and the failure is indicated by the infinite coefficients. In fact, the indefinite integral is

$$
\left(\frac{1}{4} r^{3}+\frac{5}{8} e^{2} r\right) \sqrt{ }\left(r^{2}+e^{2}\right)+\frac{3}{8} e^{4} \log \left\{r+\sqrt{ }\left(r^{2}+e^{2}\right)\right\},
$$

which between the limits is

$$
\begin{gathered}
\left(\frac{1}{4} R^{3}+\frac{5}{8} e^{2} R\right) \sqrt{ }\left(R^{2}+e^{2}\right)+\frac{3}{8} e^{4} \log \frac{R+\sqrt{ }\left(R^{2}+e^{2}\right)}{e} \\
=\frac{1}{4} R^{4}+\frac{3}{4} e^{2} R^{2}+\ldots-\frac{3}{8} e^{4} \log e .
\end{gathered}
$$

In the general case, the term causing the failure is $K e^{-2 q}$ when $q$ is fractional, and $K e^{-2 q} \log e$ when $q$ is integral. As a step towards determining the entire expansion, I notice that, writing $x=\frac{e^{2}}{e^{2}+r^{2}}$ or $r=e x^{-\frac{1}{2}}(1-x)^{\frac{1}{2}}$, the value of the integral is

where

$$
=\frac{1}{2} e^{-2 q} \int_{X}^{1} x^{q-1}(1-x)^{\frac{1}{2} s-1} d x
$$

$$
X=\frac{e^{2}}{e^{2}+R^{2}}
$$

